\title{
Relationship between Physical Activities of Women and the Prevalence of Some Common Diseases: Empirical Evidence from Saudi Arabia
}

\author{
Fawziah Al-Bakr ${ }^{1}$, Ahmed Al-Haramlah' ${ }^{2}$, Haniah Merza ${ }^{3}$ \\ ${ }^{1}$ Educational Policies Department, King Saud University, Riyadh, Saudi Arabia \\ ${ }^{2}$ Applied Sport Psychology, Northern Border University, Arar, Saudi Arabia \\ ${ }^{3}$ College of Education Director Support Center for Students with Disabilities and Accessibility Program, \\ Princess Norah University, Riyadh, Saudi Arabia \\ Email: falbaker@ksu.edu.sa, Ahmed.Alharamlah@nbu.edu.sa,Ahmad_Alharamlah@yahoo.com, \\ m.haniah@gmail.com
}

Received 26 February 2016; accepted 2 May 2016; published 6 May 2016

Copyright (C) 2016 by authors and Scientific Research Publishing Inc.

This work is licensed under the Creative Commons Attribution International License (CC BY).

http://creativecommons.org/licenses/by/4.0/

c) (i) Open Access

\begin{abstract}
The aim of this study was to explore relationships between common diseases, i.e. obesity, high blood pressure, diabetes, cholesterol as well as asthma, and socio-demographic variables: residence place, age, weight, educational level, along with level of physical activity among women in Saudi Arabia. On the strength of a purposive sample, it comprised of 1233 Saudi women from central, eastern, western, northern and southern regions of KSA. The findings supported the hypotheses that obesity, high blood pressure, diabetes, cholesterol and asthma were related to place of residence, age, weight, educational level, as well as level of physical activity. Hence, the results highlighted the importance of socio-demographic and exercise in controlling common diseases to a considerable extent.
\end{abstract}

\section{Keywords}

Physical Activity, Common Diseases, Saudi Women, Socio-Demographic Variables

\section{Introduction}

Research on common diseases gains increased attention from researchers, academics as well as clinicians either

How to cite this paper: Al-Bakr, F., Al-Haramlah, A., \& Merza, H. (2016). Relationship between Physical Activities of Women and the Prevalence of Some Common Diseases: Empirical Evidence from Saudi Arabia. Advances in Physical Education, 6, 67-75. http://dx.doi.org/10.4236/ape.2016.62008 
in developed or developing countries due to the prevalence of such diseases, which in turn results in various public health problems. A considerable body of research was undertaken to investigate risk factors related to common diseases. In one research vein, the relationship between common diseases and physical activity was investigated. Accordingly, physical activity has been deemed as a major risk factor, to which numerous diseases, e.g. cardiovascular disease, some cancers, diabetes, obesity, metabolic syndrome, coronary heart disease, high cholesterol level, osteoporosis, gallbladder disease, blood pressure, are traced back (Al-Nuaim, 1997; Al-Hazzaa, 2004; Al-Hazzaa, 2007; Bauman et al., 2009; Ng et al., 2011; Al-Nuaim et al., 2012; Al-Baker et al., 2013; Galan et al., 2013; Assah et al., 2015; Al-Haramlah, et al., 2015). According to Fox (2000), physical activity not only affects physical health but also mental health. Furthermore, mortality was documented as an outcome of physical inactivity (Aucella et al., 2014; Assah et al., 2015). Another vein of research tackled the prevalence of common diseases in different countries as well as relationships between these diseases and socio-demographic variables (Al-Rukban, 2003; Al-Daghri et al., 2011; Ng et al., 2011; Al-Baker et al., 2013; Aucella et al., 2014; Assah et al., 2015). In the Kingdom of Saudi Arabia (KSA), numerous studies have been conducted to explore prevalence of common diseases among Saudis. Examples of Saudi works included those aimed at determining the prevalence of obesity and overweight (Farghaly et al., 2007; Al-Hazzaa \& Al-Rasheedi, 2007; Al-Baghli et al., 2008; Al-Nuaim et al., 2012), metabolic syndrome (Al-Nozha et al., 2005), hypertension (Al-Nozha et al., 2007), hyperlipidemia (Al-Nozha et al., 2008), diabetes mellitus (Al-Daghri et al., 2011). However, the association between a set of common diseases, i.e., obesity, high blood pressure, diabetes, cholesterol and asthma, and different socio-demographic variables, e.g., place of residence, age group, weight, educational level in addition to physical activation a sample covers central, eastern, western, northern and southern regions of KSA was not investigated yet. Hence, the main aim of this study is to fill this gap. This paper is structured as follows. Section 2 presents related literature and hypotheses development. Section 3 illustrates research methodology. Section 4 exhibits data analysis and results. Section 5 demonstrates discussion of results and conclusion. Finally, section 6 declares implication and recommendations of the study.

\section{Literature Review and Hypotheses Development}

A center of interest for many studies conducted on societal health was the prevalence of common diseases and the relationship between these diseases and other variables such as socio-demographic variables and lifestyle behaviors. On the association between common diseases and place of residence, a recent study carried out by Assah et al. (2015) revealed a significant correlation between physical activity and place of residence. That is, rural subjects were more active and less sedentary than urban subjects. According to their results, physical inactivity was inversely related to variables such as age, educational level and obesity. It was concluded that obesity is related to place of residence. An earlier study performed by Rasheed (1998) on Saudi women from Al-Khobar with the aim of examining their behaviors of eating and physical activity as well as their perceptions of body size, accepted the hypothesis that obesity is related to eating and exercise behaviors. Al-Nozha et al. (2004) studied the prevalence of coronary artery disease in Saudi Arabia. Based on a sample consisted of 944 respondents aged between 30 - 70 years, their results pointed out a high prevalence percentage of coronary artery disease among urban Saudis in comparison with rural Saudis. Al-Nuaim et al. (2012) confirmed that the prevalence of obesity and overweight was attributed to regional differences. On the basis of the above literature, the following hypothesis was proposed: Common diseases among Saudi women are positively related to their place of residence (hypothesis 1). Concerning the relationship between common diseases and age, Al-Nozha et al., (2004) asserted that the prevalence of coronary artery disease is related to age. In their study on the influence on socio-demographic characteristics and cardiovascular factors related to prevalence of obesity, Al-Baghli et al. (2008) concluded that the highest percentage of obesity was among age group (50 - 59). According to Al-Daghri et al. (2011), there were significant differences in the prevalence of diabetes mellitus type 2 due to gender. Consequently, the following hypothesis was suggested: Common diseases among Saudi women are positively related to their age (hypothesis 2). In relation to association between common diseases and weight, it was concluded that the prevalence of hypertension is related to the weight of both gender in KSA (Al-Nozha et al., 2004). Al-Refaee and Al-Hazzaa (2001) indicated that the prevalence of obesity measured by body mass index is higher among inactive subjects. On the other hand, Khalili et al. (2013) highlighted that body mass index was not related to Ulcerative colitis and Crohn's disease. Hence, the following hypothesis was presumed: Common diseases among Saudi women are positively related to their weight (hypothesis 3). Respecting the correlation 
between common diseases and educational level, Rasheed (1998) found a significant correlation among estimation of body weight and educational level. According to Al-Baghli et al. (2008) who studied overweight and obesity in the eastern province of Saudi Arabia, less educated women were more subjected to obesity. Therefore, the following hypothesis was suggested: Common diseases among Saudi women are positively related to their educational level (hypothesis 4). Regarding the correlation between common diseases and physical activity (Assah et al., 2015), obesity was negatively related to physical activity. Ng et al. (2011) undertaken a cross-sectional study in the United Arab Emirates and concluded that 65\% of Emirati women overweight or obese. They further added that $58.8 \%$ of Emirates women have low level of physical activity. Additionally, Al-Rukban (2003) found a significant relationship between physical inactivity and prevalence of obesity among Saudi male adolescents. Thus, the following hypothesis was assumed: Common diseases among Saudi women are positively related to their level of physical activity (hypothesis 5).

\section{Research Methodology}

\subsection{Participants and Data Collection}

A total of 1500 women from different regions in Saudi Arabia comprised the sample of this study. The surveybased questionnaire adopted in this study was completed by 1327 women. Due to invalid questionnaires, 94 questionnaires were excluded. Hence, the total number of participants was 1233 women. Participants' profile can be seen in Table 1 . According to the table, $62.6 \%$ (772) of study participants were resident in the middle

\section{Table 1. Participants’ demographic characteristics.}

\begin{tabular}{|c|c|c|c|c|}
\hline Var. & Residence & Age & Education & Weight \\
\hline \multicolumn{5}{|l|}{ Place of residence } \\
\hline Middle & $772(62.6 \%)$ & & & \\
\hline Eastern & $65(5.3 \%)$ & & & \\
\hline Western & 95 (7.7\%) & & & \\
\hline Northern & $46(3.7 \%)$ & & & \\
\hline Southern & 255 (20.7\%) & & & \\
\hline \multicolumn{5}{|l|}{ Age } \\
\hline $18-25$ & & 892 (72.3\%) & & \\
\hline $26-33$ & & 155 (12.6\%) & & \\
\hline $34-41$ & & $104(8.4 \%)$ & & \\
\hline $42-51$ & & 65 (5.3\%) & & \\
\hline 52 or more & & $17(1.4 \%)$ & & \\
\hline \multicolumn{5}{|l|}{ Educational level } \\
\hline Uneducated & & & $6(0.5 \%)$ & \\
\hline Read but don not write & & & $14(1.1 \%)$ & \\
\hline Primary & & & $21(1.7 \%)$ & \\
\hline Medium & & & $3(0.2 \%)$ & \\
\hline Secondary & & & $187(15.2 \%)$ & \\
\hline Bachelor & & & $971(78.8 \%)$ & \\
\hline Master & & & $29(2.4 \%)$ & \\
\hline Doctorate & & & $2(0.2 \%)$ & \\
\hline \multicolumn{5}{|l|}{ Weight } \\
\hline $45-50$ & & & & 303 (24.6\%) \\
\hline $51-56$ & & & & $265(21.5 \%)$ \\
\hline $57-62$ & & & & $222(18.0 \%)$ \\
\hline $63-68$ & & & & $144(11.7 \%)$ \\
\hline $69-74$ & & & & 112 (9.1\%) \\
\hline $75-79$ & & & & $141(11.4 \%)$ \\
\hline 80 or more & & & & 46 (3.7\%) \\
\hline Total & 1233 & 1233 & 1233 & 1233 \\
\hline
\end{tabular}


region of KSA and $20.7 \%$ (255) were resident in the southern region. Of the participants, $72.3 \%$ were aged between 18 - 25 years, 12.6\% were between 26 - 33 years, 8.4\% (104) were between 34 - 41 years, and 5.3\% (65) were between 42 - 51 years. Finally, the percentage of participants whom age 52 years or above was $1.4 \%$ (17). The majority of the participants were held a bachelor degree $(78.8 \%, 971)$ followed by participants held secondary degree $(15.2 \%, 187)$. In terms of weight, the percentage of participants whom weight ranged between 45 and $50 \mathrm{KG}$ was $24.6 \%$ (303), 21.5\% (265) from 51 to 56 KG, 18.0\% (222) between 57 and 62 KG.

\subsection{Measures and Analysis Procedures}

A questionnaire-based survey was employed based on Al-Baker et al. (2013) to explore the relationship between common diseases (obesity, high blood pressure, diabetes, cholesterol, asthma) and other variables such as place of residence, age, educational level, weight and physical activity. The questionnaire consisted of two main parts: the demographic characteristics part, which intends to collect data on personal characteristics such as place of residence, age, educational level, weight as well as physical activity. The physical activity of a participant was measured on the basis of frequency and duration of the physical activity. The second part of the questionnaire measures common diseases such as obesity, high blood pressure, diabetes, cholesterol, asthma. Obesity was measured by asking participants to report their weight in kilograms and height in squared meter order to calculate obesity. Other constructs were measured by asking participants to indicate their level of hypertension, diabetes, cholesterol, and asthma on none, temporary, chronic categories. Additionally, participants required to mention any medications related to theses common diseases. The Statistical Package for Social Sciences (SPSS) was used for data analysis purpose.

\section{Results and Discussion}

Frequencies, percentages as well as Phi correlation factor were calculated in order to explore the relationship between common diseases prevalent among Saudi women and their place of residence as suggested in hypothesis 1 . The findings shown in Table 2 indicated significant relationships between obesity $(\mathrm{r} \varphi=0.218, \mathrm{P} \leq 0.05)$, high blood pressure $(\mathrm{r} \varphi=0.238, \mathrm{P} \leq 0.05)$, diabetes $(\mathrm{r} \varphi=0.222, \mathrm{P} \leq 0.05)$, cholesterol $(\mathrm{r} \varphi=0.210, \mathrm{P} \leq 0.05)$ along with asthma $(0.192, \mathrm{P} \leq 0.05)$ and place of residence. Furthermore, compared to women resident in other regions of KSA, women resident in the eastern region had higher percentages of obesity (42.5\%), blood pressure,

Table 2. Common diseases and place of residence.

\begin{tabular}{|c|c|c|c|c|c|c|c|c|c|c|c|}
\hline & & \multicolumn{2}{|c|}{ Obesity } & \multicolumn{2}{|c|}{ High blood pressure } & \multicolumn{2}{|c|}{ Diabetes } & \multicolumn{2}{|c|}{ Cholesterol } & \multicolumn{2}{|c|}{ Asthma } \\
\hline & & IN & UN & IN & UN & IN & UN & IN & UN & IN & UN \\
\hline \multirow[t]{2}{*}{ M } & F & 168 & 604 & 127 & 645 & 129 & 643 & 135 & 637 & 153 & 619 \\
\hline & $\%$ & 21.8 & 78.2 & 16.5 & 83.5 & 16.7 & 83.3 & 17.5 & 82.5 & 19.8 & 80.2 \\
\hline \multirow[t]{2}{*}{$\mathrm{E}$} & $\mathrm{F}$ & 27 & 38 & 25 & 40 & 25 & 40 & 25 & 40 & 25 & 40 \\
\hline & $\%$ & 41.5 & 58.5 & 38.5 & 61.5 & 38.5 & 61.5 & 38.5 & 61.5 & 38.5 & 61.5 \\
\hline \multirow[t]{2}{*}{$\mathrm{W}$} & F & 10 & 85 & 6 & 89 & 6 & 89 & 6 & 89 & 9 & 86 \\
\hline & $\%$ & 10.5 & 89.5 & 6.3 & 93.7 & 6.3 & 93.7 & 6.3 & 93.7 & 9.5 & 90.5 \\
\hline \multirow[t]{2}{*}{$\mathrm{N}$} & $\mathrm{F}$ & 0 & 46 & 0 & 46 & 0 & 46 & 0 & 46 & 0 & 46 \\
\hline & $\%$ & 0 & 100 & 0 & 100 & 0 & 100 & 0 & 100 & 0 & 100 \\
\hline \multirow[t]{2}{*}{$S$} & F & 93 & 162 & 84 & 171 & 78 & 177 & 75 & 180 & 78 & 177 \\
\hline & $\%$ & 36.5 & 63.5 & 32.9 & 67.1 & 30.6 & 69.4 & 29.4 & 70.6 & 30.6 & 69.4 \\
\hline \multirow[t]{2}{*}{$\mathrm{T}$} & F & 298 & 935 & 242 & 991 & 238 & 995 & 241 & 992 & 265 & 968 \\
\hline & $\%$ & 24.2 & 75.8 & 19.6 & 80.4 & 19.3 & 80.7 & 19.5 & 80.5 & 21.5 & 78.5 \\
\hline $\mathrm{r} \varphi$ & & \multicolumn{2}{|c|}{0.218} & \multicolumn{2}{|c|}{0.238} & \multicolumn{2}{|c|}{0.222} & \multicolumn{2}{|c|}{0.210} & \multicolumn{2}{|c|}{0.192} \\
\hline $\mathrm{P}$ & & \multicolumn{2}{|c|}{$0.000^{*}$} & \multicolumn{2}{|c|}{$0.000 *$} & \multicolumn{2}{|c|}{$0.000 *$} & \multicolumn{2}{|c|}{$0.000 *$} & \multicolumn{2}{|c|}{$0.000^{*}$} \\
\hline
\end{tabular}

M: middle region, E: eastern region, W: western region, N: northern region, S: southern region, IN: Infected, UN: Uninfected, T: total, r $\varphi$ : phi correlation, P: significance level ( $\mathrm{p}<0.05)$. 
diabetes, cholesterol and asthma (38.5\%). Hence, hypothesis 1 was accepted. According to Al-Nozha et al. (2004), there is a regional variation between urban and rural Saudis in terms of prevalence of coronary artery disease. Similarly, Al-Nuaim et al. (2012) found significant differences among Saudi subjects in terms of obesity prevalence.

Hypothesis 2 presumed a significant relationship between common diseases prevalent among Saudi women and their age. On the strength of the findings shown in Table 3, the hypothesis was supported.

According to the results, phi correlation for obesity, high blood pressure, diabetes, cholesterol and asthma ( $\mathrm{r} \varphi$ $=0.214,0.204,0.229,0.186)$ respectively. Moreover, it was revealed that obesity $(58.5 \%)$, cholesterol $(46.2 \%)$ and asthma (46.2\%) were more prevalent among Saudi women aged between 42 - 51 years. In contrast, high blood pressure (52.9\%) and diabetes (64.7\%) were more prevalent among Saudi women aged 52 years or above. This may be because the reduced level of physical activity during this age, in addition to pregnancy, childbirth as well aging outcomes. Similar results were echoed by Al-Nozha et al. (2004) who found an association between the prevalence of coronary artery disease and age, and Al-Baghli et al. (2008) who linked the prevalence of obesity to participants' age group.

Hypothesis 3 suggested a significant association between common diseases prevalent among Saudi women and their weight. Frequencies, percentages and Phi correlation factor were calculated for the purpose of testing this hypothesis. Based on the results depicted in Table 4, there was a significant relationship between obesity $(\mathrm{r} \varphi=0.315, \mathrm{P} \leq 0.05)$, high blood pressure $(\mathrm{r} \varphi=0.277, \mathrm{P} \leq 0.05)$, diabetes $(\mathrm{r} \varphi=0.285, \mathrm{P} \leq 0.05)$, cholesterol $(\mathrm{r} \varphi=0.242, \mathrm{P} \leq 0.05)$ along with asthma $(\mathrm{r} \varphi=0.236, \mathrm{P} \leq 0.05)$ and weight. The results further highlighted that the prevalence of obesity (54.3\%), high blood pressure (50\%), diabetes (43.5\%), cholesterol (43.5\%) and asthma (37\%). These results may attribute to unhealthy eating habits and the low level of physical activity. In their 2004 study, Al-Nozha et al. found a relationship between hypertension prevalence and participants' weight.

Hypothesis 4 assumed that common diseases prevalent among Saudi women were significantly related to educational level. It was found on the basis of data analysis, as shown in Table 5, that obesity ( $\mathrm{r} \varphi=0.204, \mathrm{P} \leq$ $0.05)$, high blood pressure ( $\mathrm{r} \varphi=0.163, \mathrm{P} \leq 0.05)$, diabetes $(\mathrm{r} \varphi=0.188, \mathrm{P} \leq 0.05)$, cholesterol $(\mathrm{r} \varphi=0.177, \mathrm{P} \leq$ 0.05 ) along with asthma ( $r \varphi=0.206, P \leq 0.05)$ were associated to the educational level of Saudi women. In the same line, the results pointed out that Saudi women who held a medium level of education have the highest percentage of obesity, high blood pressure, diabetes, cholesterol and asthma (100\%). This may due to the medium level of health awareness as well as lessened perceptions towards the significance of the role played by physical activity in curbing the prevalence of common diseases. Al-Baghli et al. (2008) stated that overweight and obesity in the eastern province of Saudi Arabia, were related to educational level of Saudi women.

Table 3. Common diseases and age.

\begin{tabular}{|c|c|c|c|c|c|c|c|c|c|c|c|}
\hline & & \multicolumn{2}{|c|}{ Obesity } & \multicolumn{2}{|c|}{ High blood pressure } & \multicolumn{2}{|c|}{ Diabetes } & \multicolumn{2}{|c|}{ Cholesterol } & \multicolumn{2}{|c|}{ Asthma } \\
\hline & & IN & UN & IN & UN & IN & UN & IN & UN & IN & UN \\
\hline \multirow[t]{2}{*}{1} & $\mathrm{~F}$ & 177 & 715 & 143 & 749 & 137 & 755 & 143 & 749 & 162 & 730 \\
\hline & $\%$ & 19.8 & 80.2 & 16.0 & 84.0 & 15.4 & 84.6 & 16.0 & 84.0 & 18.2 & 81.8 \\
\hline \multirow[t]{2}{*}{2} & $\mathrm{~F}$ & 45 & 110 & 33 & 122 & 33 & 122 & 35 & 120 & 40 & 115 \\
\hline & $\%$ & 29.0 & 71.0 & 21.3 & 78.7 & 21.3 & 78.7 & 22.6 & 77.4 & 25.8 & 74.2 \\
\hline \multirow[t]{2}{*}{3} & $\mathrm{~F}$ & 32 & 72 & 27 & 77 & 27 & 77 & 27 & 77 & 27 & 77 \\
\hline & $\%$ & 30.8 & 69.2 & 26.0 & 74.0 & 26.0 & 74.0 & 26.0 & 74.0 & 26.0 & 74.0 \\
\hline \multirow[t]{2}{*}{4} & $\mathrm{~F}$ & 38 & 27 & 30 & 35 & 30 & 35 & 30 & 35 & 30 & 35 \\
\hline & $\%$ & 58.5 & 41.5 & 46.2 & 53.8 & 46.2 & 53.8 & 46.2 & 53.8 & 46.2 & 53.8 \\
\hline \multirow[t]{2}{*}{5} & $\mathrm{~F}$ & 6 & 11 & 9 & 8 & 11 & 6 & 6 & 11 & 6 & 11 \\
\hline & $\%$ & 35.3 & 64.7 & 52.9 & 47.1 & 64.7 & 35.3 & 35.3 & 64.7 & 35.3 & 64.7 \\
\hline \multirow[t]{2}{*}{$\mathrm{T}$} & $\mathrm{F}$ & 298 & 935 & 242 & 991 & 238 & 995 & 241 & 992 & 265 & 968 \\
\hline & $\%$ & 24.2 & 75.8 & 19.6 & 80.4 & 19.3 & 80.7 & 19.5 & 80.5 & 21.5 & 78.5 \\
\hline $\mathrm{r} \varphi$ & & \multicolumn{2}{|c|}{0.214} & \multicolumn{2}{|c|}{0.204} & \multicolumn{2}{|c|}{0.229} & \multicolumn{2}{|c|}{0.186} & \multicolumn{2}{|c|}{0.166} \\
\hline $\mathrm{P}$ & & \multicolumn{2}{|c|}{$0.000 *$} & \multicolumn{2}{|c|}{$0.000 *$} & \multicolumn{2}{|c|}{$0.000 *$} & \multicolumn{2}{|c|}{$0.000 *$} & \multicolumn{2}{|c|}{$0.000 *$} \\
\hline
\end{tabular}

1: 18 - 25, 2: 26 - 33, 3: 34 - 41, 4: 42 - 51, 5: 52 or above, IN: Infected, UN: Uninfected, T: total, r $\varphi$ : phi correlation, P: significance level (p < 0.05). 
Table 4. Common diseases and weight.

\begin{tabular}{|c|c|c|c|c|c|c|c|c|c|c|c|}
\hline & & \multicolumn{2}{|c|}{ Obesity } & \multicolumn{2}{|c|}{ High blood pressure } & \multicolumn{2}{|c|}{ Diabetes } & \multicolumn{2}{|c|}{ Cholesterol } & \multicolumn{2}{|c|}{ Asthma } \\
\hline & & IN & $\mathrm{UN}$ & IN & UN & IN & $\mathrm{UN}$ & IN & UN & IN & UN \\
\hline \multirow[t]{2}{*}{1} & $\mathrm{~F}$ & 21 & 282 & 18 & 285 & 18 & 285 & 21 & 282 & 26 & 277 \\
\hline & $\%$ & 6.9 & 93.1 & 5.9 & 94.1 & 5.9 & 94.1 & 6.9 & 93.1 & 8.6 & 91.4 \\
\hline \multirow[t]{2}{*}{2} & $\mathrm{~F}$ & 56 & 209 & 51 & 214 & 51 & 214 & 53 & 212 & 53 & 212 \\
\hline & $\%$ & 21.1 & 78.9 & 19.2 & 80.8 & 19.2 & 80.8 & 20.0 & 80.0 & 20.0 & 80.0 \\
\hline \multirow[t]{2}{*}{3} & $\mathrm{~F}$ & 44 & 178 & 34 & 188 & 34 & 188 & 37 & 185 & 42 & 180 \\
\hline & $\%$ & 19.8 & 80.2 & 15.3 & 84.7 & 15.3 & 84.7 & 16.7 & 83.3 & 18.9 & 81.1 \\
\hline \multirow[t]{2}{*}{4} & $\mathrm{~F}$ & 48 & 96 & 40 & 104 & 42 & 102 & 40 & 104 & 42 & 102 \\
\hline & $\%$ & 33.3 & 66.7 & 27.8 & 72.2 & 29.2 & 70.8 & 27.8 & 72.2 & 29.2 & 70.8 \\
\hline \multirow[t]{2}{*}{5} & $\mathrm{~F}$ & 49 & 63 & 26 & 86 & 26 & 86 & 23 & 89 & 31 & 81 \\
\hline & $\%$ & 43.8 & 56.3 & 23.2 & 76.8 & 23.2 & 76.8 & 20.5 & 79.5 & 27.7 & 72.3 \\
\hline \multirow[t]{2}{*}{6} & $\mathrm{~F}$ & 55 & 86 & 50 & 91 & 47 & 94 & 47 & 94 & 54 & 87 \\
\hline & $\%$ & 39.0 & 61.0 & 35.5 & 64.5 & 33.3 & 66.7 & 33.3 & 66.7 & 38.3 & 61.7 \\
\hline \multirow[t]{2}{*}{7} & $\mathrm{~F}$ & 25 & 21 & 23 & 23 & 20 & 26 & 20 & 26 & 17 & 29 \\
\hline & $\%$ & 54.3 & 45.7 & 50.0 & 50.0 & 43.5 & 56.5 & 43.5 & 56.5 & 37.0 & 63.0 \\
\hline \multirow[t]{2}{*}{$\mathrm{T}$} & $\mathrm{F}$ & 298 & 935 & 242 & 991 & 238 & 995 & 241 & 992 & 265 & 968 \\
\hline & $\%$ & 24.2 & 75.8 & 19.6 & 80.4 & 19.3 & 80.7 & 19.5 & 80.5 & 21.5 & 78.5 \\
\hline $\mathrm{r} \varphi$ & & \multicolumn{2}{|c|}{0.315} & \multicolumn{2}{|c|}{0.277} & \multicolumn{2}{|c|}{0.285} & \multicolumn{2}{|c|}{0.242} & \multicolumn{2}{|c|}{0.236} \\
\hline $\mathrm{P}$ & & \multicolumn{2}{|c|}{$0.000 *$} & \multicolumn{2}{|c|}{$0.000 *$} & \multicolumn{2}{|c|}{$0.000 *$} & \multicolumn{2}{|c|}{$0.000 *$} & \multicolumn{2}{|c|}{$0.000 *$} \\
\hline
\end{tabular}

1: 45 - 50, 2: 51 - 56, 3: 57 - 62, 4: 63 - 68, 5: 69 - 74, 6: 75 - 79, 7: 80 or more. IN: Infected, UN: Uninfected, T: total, r $\varphi$ : phi correlation, P: significance level $(\mathrm{p}<0.05)$.

Table 5. Common diseases and educational level.

\begin{tabular}{|c|c|c|c|c|c|c|c|c|c|c|c|}
\hline & & \multicolumn{2}{|c|}{ Obesity } & \multicolumn{2}{|c|}{ High blood pressure } & \multicolumn{2}{|c|}{ Diabetes } & \multicolumn{2}{|c|}{ Cholesterol } & \multicolumn{2}{|c|}{ Asthma } \\
\hline & & IN & UN & IN & UN & IN & UN & IN & UN & IN & UN \\
\hline \multirow[t]{2}{*}{1} & $\mathrm{~F}$ & 3 & 3 & 3 & 3 & 0 & 6 & 0 & 6 & 0 & 6 \\
\hline & & 50.0 & 50.0 & 50.0 & 50.0 & .0 & 100 & .0 & 100 & .0 & 100 \\
\hline \multirow[t]{2}{*}{2} & $\mathrm{~F}$ & 8 & 6 & 5 & 9 & 8 & 6 & 5 & 9 & 8 & 6 \\
\hline & & 57.1 & 42.9 & 35.7 & 64.3 & 57.1 & 42.9 & 35.7 & 64.3 & 57.1 & 42.9 \\
\hline \multirow[t]{2}{*}{3} & $\mathrm{~F}$ & 0 & 21 & 3 & 18 & 3 & 18 & 3 & 18 & 0 & 21 \\
\hline & & 0 & 100 & 14.3 & 85.7 & 14.3 & 85.7 & 14.3 & 85.7 & 0 & 100 \\
\hline \multirow[t]{2}{*}{4} & $\mathrm{~F}$ & 3 & 0 & 0 & 3 & 0 & 3 & 0 & 3 & 0 & 3 \\
\hline & & 100 & 0 & 0 & 100 & 0 & 100 & 0 & 100 & 0 & 100 \\
\hline \multirow[t]{2}{*}{5} & $\mathrm{~F}$ & 71 & 116 & 61 & 126 & 61 & 126 & 63 & 124 & 67 & 120 \\
\hline & & 38.0 & 62.0 & 32.6 & 67.4 & 32.6 & 67.4 & 33.7 & 66.3 & 35.8 & 64.2 \\
\hline \multirow[t]{2}{*}{6} & $\mathrm{~F}$ & 208 & 763 & 167 & 804 & 164 & 807 & 170 & 801 & 190 & 781 \\
\hline & & 21.4 & 78.6 & 17.2 & 82.8 & 16.9 & 83.1 & 17.5 & 82.5 & 19.6 & 80.4 \\
\hline \multirow[t]{2}{*}{7} & $\mathrm{~F}$ & 5 & 24 & 3 & 26 & 2 & 27 & 0 & 29 & 0 & 29 \\
\hline & & 17.2 & 82.8 & 10.3 & 89.7 & 6.9 & 93.1 & 0 & 100 & 0 & 100 \\
\hline \multirow[t]{2}{*}{8} & F & 0 & 2 & 0 & 2 & 0 & 2 & 0 & 2 & 0 & 2 \\
\hline & & 0 & 100 & & 100 & 0 & 100 & 0 & 100 & 0 & 100 \\
\hline \multirow[t]{2}{*}{$\mathrm{T}$} & $\mathrm{F}$ & 298 & 935 & 242 & 991 & 238 & 995 & 241 & 992 & 265 & 968 \\
\hline & & 24.2 & 75.8 & 19.6 & 80.4 & 19.3 & 80.7 & 19.5 & 80.5 & 21.5 & 78.5 \\
\hline $\mathrm{r} \varphi$ & & \multicolumn{2}{|c|}{0.204} & \multicolumn{2}{|c|}{0.163} & \multicolumn{2}{|c|}{0.188} & \multicolumn{2}{|c|}{0.177} & \multicolumn{2}{|c|}{0.206} \\
\hline $\mathrm{P}$ & & \multicolumn{2}{|c|}{$0.000 *$} & \multicolumn{2}{|c|}{$0.000^{*}$} & \multicolumn{2}{|c|}{$0.000^{*}$} & \multicolumn{2}{|c|}{$0.000^{*}$} & \multicolumn{2}{|c|}{$0.000 *$} \\
\hline
\end{tabular}

1: Uneducated, 2: Read but don write, 3: Primary, 4: Medium, 5: Secondary, 6: Bachelor, 7: Master, 8: Doctorate, IN: Infected, UN: Uninfected, T: total, $\mathrm{r} \varphi$ : phi correlation, $\mathrm{P}$ : significance level $(\mathrm{p}<0.05)$. 
Hypothesis 5 postulated that common diseases prevalent among Saudi women were significantly linked to physical activity. The results presented in Table 6 clarified a significant relationship between asthma $(\mathrm{r} \varphi=$ $0.080, \mathrm{P} \leq 0.05)$ and physical activity. Contrary, no significant relationships found between obesity $(\mathrm{r} \varphi=0.032$, $\mathrm{P}>0.05)$, high blood pressure $(\mathrm{r} \varphi=0.045, \mathrm{P}>0.05)$, diabetes $(\mathrm{r} \varphi=0.053, \mathrm{P}>0.05)$, cholesterol $(\mathrm{r} \varphi=0.036, \mathrm{P}>$ 0.05) and physical activity. Similar findings were found in different countries. In Cameron, Assah et al. (2015) linked obesity to physical inactivity. In the United Arab Emirates, Ng et al. (2011) asserted the same result, where $65 \%$ of Emirati women were obese or overweight with a low level of physical activity. In KSA, Al-Rukban (2003) found a significant association between physical inactivity and prevalence of obesity among Saudi male adolescents.

\section{Conclusion}

The aim of this study was to investigate relationships between common diseases, i.e. obesity, high blood pressure, diabetes, cholesterol and asthma, and socio-demographic variables: residence place, age, weight, educational level, as well as level of physical activity among women in Saudi Arabia. The results revealed a significant relationship between all common diseases studied and place of residence. Particularly, the highest prevalence of common diseases was observed among women resident in the eastern region. In terms of age, there was a signification relationship between all common diseases explored in this study and women age. The prevalence of common diseases was higher among women aged 42 to 51 years old. Equally, weight and educational level were related to common diseases, the prevalence of common diseases was higher among women aged $80 \mathrm{KG}$ or more and women held medium educational level. Finally, physical activity was linked to asthma only. No relationships were found between obesity, high blood pressure, diabetes, as well as cholesterol and physical activity. Consequently, the study concluded that the prevalence of common diseases studies, i.e. obesity, high blood pressure, diabetes, cholesterol and asthma was related to residence place, age, weight, and educational level, but not to physical activity among women in Saudi Arabia. However, asthma was only associated to physical activity.

\section{Limitations, Recommendations and Future Research}

One of the most critical limitations of the study was the accurate measurements of variables such as obesity, high blood pressure, diabetes, cholesterol, along with asthma. The significance of socio-demographic variables was regarded in this study as critical factors affected the prevalence of common diseases among Saudi women. Accordingly, we recommend that physical activities should be allowed in female schools as to get girls accustomed to it in a regular base as Saudi women up to now are not allowed to do any sport activities in public schools. As evident by our findings opening of sport facilities in all local communities that women can easily reach by foot. Socio-demographic variables as evident by our findings should be taken in consideration when

Table 6. Common diseases and physical activity.

\begin{tabular}{|c|c|c|c|c|c|c|c|c|c|c|c|}
\hline & & \multicolumn{2}{|c|}{ Obesity } & \multicolumn{2}{|c|}{ High blood pressure } & \multicolumn{2}{|c|}{ Diabetes } & \multicolumn{2}{|c|}{ Cholesterol } & \multicolumn{2}{|c|}{ Asthma } \\
\hline & & IN & UN & IN & UN & IN & UN & IN & UN & IN & UN \\
\hline \multirow[t]{2}{*}{1} & $\mathrm{~F}$ & 61 & 213 & 47 & 227 & 44 & 230 & 49 & 225 & 43 & 231 \\
\hline & $\%$ & 22.3 & 77.7 & 17.2 & 82.8 & 16.1 & 83.9 & 17.9 & 82.1 & 15.7 & 84.3 \\
\hline \multirow[t]{2}{*}{2} & $\mathrm{~F}$ & 202 & 600 & 168 & 634 & 167 & 635 & 165 & 637 & 190 & 612 \\
\hline & $\%$ & 25.2 & 74.8 & 20.9 & 79.1 & 20.8 & 79.2 & 20.6 & 79.4 & 23.7 & 76.3 \\
\hline \multirow[t]{2}{*}{3} & $\mathrm{~F}$ & 35 & 122 & 27 & 130 & 27 & 130 & 27 & 130 & 32 & 125 \\
\hline & $\%$ & 22.3 & 77.7 & 17.2 & 82.8 & 17.2 & 82.8 & 17.2 & 82.8 & 20.4 & 79.6 \\
\hline \multirow[t]{2}{*}{$\mathrm{T}$} & $\mathrm{F}$ & 298 & 935 & 242 & 991 & 238 & 995 & 241 & 992 & 265 & 968 \\
\hline & $\%$ & 24.2 & 75.8 & 19.6 & 80.4 & 19.3 & 80.7 & 19.5 & 80.5 & 21.5 & 78.5 \\
\hline $\mathrm{r} \varphi$ & & \multicolumn{2}{|c|}{0.032} & \multicolumn{2}{|c|}{0.045} & \multicolumn{2}{|c|}{0.053} & \multicolumn{2}{|c|}{0.036} & \multicolumn{2}{|c|}{0.080} \\
\hline $\mathrm{P}$ & & \multicolumn{2}{|c|}{0.523} & \multicolumn{2}{|c|}{0.281} & \multicolumn{2}{|c|}{0.175} & \multicolumn{2}{|c|}{0.456} & \multicolumn{2}{|c|}{$0.020^{*}$} \\
\hline
\end{tabular}

1: No, 2: Occasionally, 3: Yes, IN: Infected, UN: Uninfected, T: total, r $\varphi$ : phi correlation, P: significance level ( $\mathrm{p}<0.05)$. 
studying the prevalence of any and other research findings that region is significantly related to these common diseases, we recommend the of common diseases. Further studies should be conducted to verify the findings of this study, particularly, to explore the relationship between prevalence of common diseases and physical activity.

\section{Acknowledgements}

This research project was supported by a grant from The Research Center for the Humanities, Deanship of Scientific Research at King Saud University, Riyadh, Saudi Arabia.

\section{References}

Al-Baghli, N., Al-Ghamdi, A., Al-Turki, K., El-Zubaier, A., Al-Ameer, M., \& Al-Baghli, F. (2008). Overweight and Obesity in the Eastern Province of Saudi Arabia. Saudi Medical Journal, 29, 1319-1325.

Al-Baker, F., Al-Haramlah, A., \& Mreza, H. (2013). Saudi Women's Attitudes towards Sports Activities and Early Intervention Strategies to Reduce the Health Consequences. A Research Presented to the Scientific Conference XIII of the Science of Sports Psychology, the International Society of Sports Psychology ISSP, Beijing.

Al-Daghri, N., Al-Attas, O., Alokail, M., Alkharfy, K., Yousef, M., Sabico, S., \& Chrousos, G. (2011). Diabetes Mellitus Type 2 and Other Chronic Common Diseases in the Central Region, Saudi Arabia (Riyadh Cohort 2): A Decade of an Epidemic. BMC Medicine, 9, 1-6.

Al-Haramlah, A., Al-Bakr, F., \& Merza, H. (2015). Common Diseases and Some Demographic Characteristics among Saudi Women, International Education Studies, 8, 95-107. http://dx.doi.org/10.5539/ies.v8n12p94

Al-Hazzaa, H., \& Al-Rasheedi, A. (2007). Adiposity and Physical Activity Levels among Preschool Children in Jeddah, Saudi Arabia. Saudi Medical Journal, 28, 766-773.

Al-Hazzaa, H. (2004). Prevalence of Physical Inactivity in Saudi Arabia: A Brief Review. Eastern Mediterranean Health Journal, 10, 663-670.

Al-Hazzaa, H. (2007). Health-Enhancing Physical Activity among Saudi Adults Using the International Physical Activity Questionnaire (IPAQ). Public Health Nutrition, 10, 59-64. http://dx.doi.org/10.1017/S1368980007184299

Al-Nozha, M., Al-Maatouq, M., Al-Mazrou, Y., Al-Harthi, S., Arafah, M., Khalil, M., Khan, N., Al-Khadra, A., Al-Marzouki, K., Nouh, M., Abdullah, M., Attas, O., Al-Shahid, M., \& Al-Mobeireek, A. (2004). Diabetes Mellitus in Saudi Arabia. Saudi Medical Journal, 25, 1603-1610.

Al-Nozha, M., Al-Mazrou, Y., Al-Maatouq, M., Arafah, M., Khalil, M., Khan, N., Al-Marzouki, K., Abdullah, M., Al- Khadra, A., Al-Harthi, S., Al-Shahid, M., Al-Mobeireek, A., \& Nouh, M. (2005). Obesity in Saudi Arabia. Saudi Medical Journal, 26, 824-829.

Al-Nozha, M., Abdullah, M., Arafah, M., Khalili, M., Khan, N., Al-Mazrou, Y., Al-Maatouq, M., Al-Marzouki, K., AlKhadra, A., Nouh, M., Al-Harthi, S., Al-Shahid, M., \& Al-Mobeireek, A. (2007). Hypertension in Saudi Arabia. Saudi Medical Journal, 28, 77-84.

Al-Nozha, M., Arafah, M., Al-Maatouq, M., Khalili, M., Khan, N., Al-Marzouki, K., Al-Mazrou, Y., Abdullah, M., AlKhadra, A., Al-Harthi, S., Al-Shahid, M., Al-Mobeireek, A., \& Nouh, M. (2008). Hyperlipidemia in Saudi Arabia. Saudi Medical Journal, 29, 282-287.

Al-Nuaim, A., Al-Nakeeb, Y., Lyons, M., Al-Hazzaa, H., Nevill, A., Collins, P., \& Duncan, M. (2012). The Prevalence of Physical Activity and Sedentary Behaviours Relative to Obesity among Adolescents from Al-Ahsa, Saudi Arabia: Rural versus Urban Variations. Journal of Nutrition and Metabolism, 2012, Article ID: 417589.

http://dx.doi.org/10.1155/2012/417589

Al-Nuaim, A. (1997). Population-Based Epidemiological Study of the Prevalence of Overweight and Obesity in Saudi Arabia, Regional Variation. Saudi Medical Journal, 17, 195-199.

Al-Refaee, S., \& Al-Hazzaa, H. (2001). Physical Activity Profile of Adult Males in Riyadh City. Saudi Medical Journal, 22, 784-789.

Al-Rukban, M. (2003). Obesity among Saudi Male Adolescents in Riyadh, Saudi Arabia. Saudi Medical Journal, $24,27-33$.

Assah, F., Mbanya, J., Ekelund, U., Wareham, N., \& Brage, S. (2015). Patterns and Correlates of Objectively Measured Free-Living Physical Activity in Adults in Rural and Urban Cameroon. Journal of Epidemiology \& Community Health, 69, 700-707. http://dx.doi.org/10.1136/jech-2014-205154

Aucella, F., Valente, G., \& Catizone, L. (2014). The Role of Physical Activity in the CKD Setting. Kidney and Blood Pressure Research, 39, 97-106. http://dx.doi.org/10.1159/000355783 
Bauman, A., Bull, F., Chey, T., Craig, C., Ainsworth, B., Sallis, J., Bowles, H., Hagstromer, M., Sjostrom, M., Pratt, M., \& IPS Group (2009). The International Prevalence Study on Physical Activity: Results from 20 Countries. International Journal of Behavioral Nutrition and Physical Activity, 6, 21. http://dx.doi.org/10.1186/1479-5868-6-21

Farghaly, N., Ghazali, B., Al-Wabel, H., Sadek, A., \& Abbag, F. (2007). Life Style and Nutrition and Their Impact on Health of Saudi School Students in Abha, Southwestern Region of Saudi Arabia. Saudi Medical Journal, 28, 415-421.

Fox, K. (2000). Physical Activity and Mental Health Promotion: The Natural Partnership. Journal of Public Mental Health, 2, 4-12. http://dx.doi.org/10.1108/17465729200000002

Galan, I., Boix, R., Medrano, M., Ramos, P., Rivera, F., Pastor-Barriuso, R., \& Moreno, C. (2013). Physical Activity and Self-Reported Health Status among Adolescents: A Cross-Sectional Population-Based Study. BMJ Open, 3, e002644. http://dx.doi.org/10.1136/bmjopen-2013-002644

Khalili, H., Ananthakrishnan, A., Konijeti, G., Liao, X., Higuchi, L., Fuchs, C., Spiegelman, D., Richter, J., Korzenik, J., \& Chan, A. (2013). Physical Activity and Risk of Inflammatory Bowel Disease: Prospective Study from the Nurses' Health Study Cohorts. BMJ, 347, f6633. http://dx.doi.org/10.1136/bmj.f6633

Ng, S. W., Harriso, H., Yeatt, K., El Sadig, M., \& Popkin, B. M. (2011). Nutrition Transition in the United Arab Emirates. European Journal of Clinical Nutrition, 65, 1328-1337. http://dx.doi.org/10.1038/ejcn.2011.135

Rasheed, R. (1998). Perception of Body Weight and Self-Reported Eating and Exercise Behaviour among Obese and Non-Obese Women in Saudi Arabia. Public Health, 112, 409-414. http://dx.doi.org/10.1016/S0033-3506(98)00288-1 